\title{
Gambling Problem by Gambler Sub-Types among College Students in Korea
}

\author{
Seong-Ui Kim, Jung-Hyun Choi, Jum-Mi Park
}

\begin{abstract}
This study aims to investigate a seriousness of gambling problems by gambler sub-type among college students in Korea. Data were collected from 581 college students of Seoul, Gyeonggi-do, Chungcheong-do, and Gyeongsang-do area through the questionnaire and a total of 577 questionnaires were statistically processed excluding the questionnaires of missing answers. To analyze the gambling problems by gambler sub-types among college students, a cross-sectional research design was used in the study. Data were analyzed using Statistical Package for the Social Sciences. Among the 577 respondents of this study, $62.2 \%$ had gambling experience and especially $6.1 \%$ had illegal gambling experience. The prevalence rate of gambling addiction by the Canadian problem gambling index was $14.0 \%$ in this study. A significant statistical difference between gambler sub-types in gender, college grade, spending money, beginning of the first gambling, illegal gambling experience, route to start gambling, self-esteem, impulsivity, and irrational gambling belief was found in this study. The number of respondents who knew free counselling centers when there was a gambling problem was only $20(3.5 \%)$, so it is quite required to carry out preventive education and to publicize free counselling centers for a gambling problem. In case that the respondents have a gambling problem, what they wanted to be supported most was psychotherapy and counseling, family counseling, hospital treatment, group therapy or Gamblers Anonymous meeting, and financial and legal consultation. It is required to continue further surveys on gambling among college students and to take a proper measure preventing a gambling problem.
\end{abstract}

Keywords : Gambling problem, Gambler sub-type, CPGI, College students, Korea.

\section{INTRODUCTION}

The Gallup Korea Research Institute which was commissioned by The National Gambling Control Commission to investigate 'Actual State of Use in Gambling Industry' reported that the prevalence rate of gambling addiction in Korean was serious level. The Institute used the Canadian problem gambling index (CPGI) classified gambler sub-types as non-problem, low risk, moderate risk, and problem gambling [1,2]. The institute calculated a prevalence rate of gambling addiction as a sum of moderate risk gambling and problem gambling [3]. This study used and defined the term, 'prevalence rate of gambling addiction,' in the same way with the Gallup Korea Research Institute so that it could

Revised Manuscript Received on July 22, 2019

Seong-Ui Kim, Department of Nursing, Saekyung College, Yeongwol-eup, South Korea. Email: agape2816@ hanmail.net

*Jung-Hyun Choi, Department of Nursing, Namseoul University, Cheonan, South Korea. Email: jhc@nsu.ac.kr

Jum-Mi Park, Department of Nursing, Namseoul University, Cheonan, South Korea. Email: jump@nsu.ac.kr be compared with the existing survey data of the institute.

The prevalence rates of gambling addiction in Korean adults over 20 years of age were $7.2 \%$ in $2012,5.4 \%$ in 2014 , and $5.1 \%$ in 2016 [3] and they were much higher than the Czech Republic's 2\% of 2015 [4] which was conducted as national scale surveys using the CPGI. Meanwhile, the rate of college students in 2011 was $11.1 \%$ in Korea [5]. The National Gambling Control Commission carries out actual situation survey on gambling for adults every two years, however, there are not many researches specified to college students. Therefore, this study was focused to college students.

If a person is enjoying light fun or getting friendship with someone through legitimate gambling and there is no problem in his or her daily life, there will be no problem in gambling. However, if the frequency of gambling becomes frequent and addictive, it can harm not only himself but also family and society.

Based on the American Psychological Association (APA)'s Diagnostic and Statistical Manual of Mental Disorders, Fourth Edition (DSM-IV) [6], Lee et al. described the process of gambling as follows: if gamblers reach addictive gambling through excessive gambling or problematic gambling level, they constantly obsess on gambling, pursue stimulation and excitement, and develop resistance with an increasing amount of betting [7]. More and more gambling is difficult to control, and if they do not gamble, they experience withdrawal symptoms such as anxiety or irritability. There happens a vicious cycle in which they lie to hide their gambling behaviors and fall into drinking or gambling to avoid economic difficulties, anxiety and depression in reality. Eventually, they fail to adapt to reality, and experience financial ruin and fall $[7,8]$. Problem gambling does not cause tolerance or withdrawal symptom, however, it makes a gambler lost self-control or do excessive betting, and those things put a huge loss of money on the gambler and cause problem behaviors such as serious damage to the job function and family life $[9,10]$. This study aims to investigate a seriousness of gambling problems among college students in Korea and a relationship between several variables and gambler sub-type of CPGI.

\section{MATERIALS AND METHODS}

\section{A.Participants}

Data were collected from 581 college students of Seoul, Gyeonggi-do, Chungcheong-do, and Gyeongsang-do area through the questionnaire and a total of 577 questionnaires were statistically processed excluding

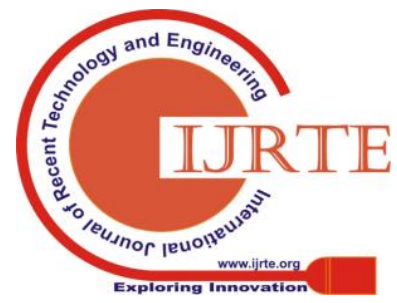


the questionnaires of missing answers.

\section{B. Research Design and Data analysis}

A cross-sectional research design was used to analyze the gambling problems by gambler sub-types among college students and Statistical Package for the Social Sciences was used to analyze the data in this study.

\section{C.Measurement}

1) Canadian problem gambling index (CPGI)

The respondents of this study answered to 9 items of CPGI [1]. Each item is evaluated with the Likert scale from 0 to 3 points $(0$ point $=$ never, 1 point $=$ sometimes, 2 points $=$ most of the time, 3 points=almost always), and scores of 9 items are combined. If combined scores are zero, one to two points, three to seven points, and more than seven points, then they are classified as non-problem, low risk, moderate risk, and problem gamblers respectively. The prevalence rate of gambling addiction includes moderate risk gamblers and problem gamblers. The Cronbach's alpha coefficient was .84.

2) Happiness

Happiness scales made by Lyubomirsky and Lepper were used in this study. It is composed of 4 items asking about a degree of subjective happiness (e.g., "Compared to my colleagues, I think I'm happy.”) [11]. Items are evaluated with 7 point Likert scale. If the score of a student is higher than other students, it means that a happiness level of the student is better than others. The Cronbach's alpha coefficient was .85.

3) Self-esteem

Self-esteem scales made by Rosenberg were used in this study. It is composed of 10 items asking about a degree of self-esteem (e.g., "I have a lot of good points.", "I am very competent person.") [12]. Items are evaluated with 4 point Likert scale. If the score of a student is higher than other students, it means that a self-esteem level of the student is better than others. The Cronbach's alpha coefficient was .78.

4) Impulsivity

Impulsivity used by Kim and Ahn was utilized [13]. It is composed of 8 items asking about an impulsivity. Items are evaluated with 4 point Likert scale. Average points of 8 items indicate a score of impulsivity. If the score of a student is higher than other students, it means that an impulsivity level of the student is higher than others. The Cronbach's alpha coefficient was .86.

5) Irrational gambling belief

In this study, irrational gambling belief scale is composed of 10 items which were selected by Kwon [14] from the items developed by Lee [15] on the basis of Steenbergh's questionnaire for gambling belief [16]. Items are evaluated with 5 point Likert scale. Average points of ten items indicate a score of irrational gambling belief. If the score of a student is higher than other students, it means that an irrational gambling belief level of the student is higher than others. The Cronbach's alpha coefficient was .92 .

\section{D.Ethical Consideration}

The college students consented to participate in this study after they received an information about a purpose, benefits and potential risks of this study, then the data were collected. It was allowed that any student could change his/her mind of participation in this study at any time.

\section{RESULTS}

\section{A.General characteristics}

Table 1 describes the general characteristics of the respondents. The average age of respondents was 20.8 years old. Among them, $45.6 \%$ were males and $54.4 \%$ were females. In the college grade, freshman was $36.4 \%$, 2nd grade was $31.2 \%$, 3rd grade was $18.7 \%$ and 4 th grade was $13.7 \%$. In residence, $20.7 \%$ lived in Seoul, $40.8 \%$ in Gyeonggi-do, $20.7 \%$ in Chungcheong-do, and $17.9 \%$ in Gyeongsang-do. The average spending money was 364,000 won per month. There were 61 students (10.6\%) who begun gambling experience at elementary school days, 73 students $(12.7 \%)$ begun at middle school days, 75 students $(13.1 \%)$ begun at high school days, and 117 students $(20.4 \%)$ begun at college days. Among the 577 respondents, $6.1 \%$ had illegal gambling experience. In the route to start gambling, $44.1 \%$ of 'suggestion from friends' was the most common response.

Table- I: General characteristics $\quad(\mathrm{N}=577)$

\begin{tabular}{|c|c|c|}
\hline Variable & Category & $\begin{array}{c}\text { Mean } \pm \text { SD, N } \\
(\%)\end{array}$ \\
\hline Age & - & $20.8 \pm 2.5$ \\
\hline \multirow{2}{*}{ Gender } & Male & $263(45.6 \%)$ \\
\hline & Female & $314(54.4 \%)$ \\
\hline \multirow{4}{*}{$\begin{array}{l}\text { College } \\
\text { grade }\end{array}$} & Freshman & $210(36.4 \%)$ \\
\hline & $2^{\text {nd }}$ grade & $180(31.2 \%)$ \\
\hline & $3^{\text {rd }}$ grade & $108(18.7 \%)$ \\
\hline & $4^{\text {th }}$ grade & $79(13.7 \%)$ \\
\hline \multirow{4}{*}{ Residence } & Seoul & $119(20.7 \%)$ \\
\hline & Gyeonggi-do & $235(40.8 \%)$ \\
\hline & Chungcheong -do & $119(20.7 \%)$ \\
\hline & Gyeongsang-do & $103(17.9 \%)$ \\
\hline \multirow{3}{*}{$\begin{array}{l}\text { Spending } \\
\text { money per } \\
\text { month }\end{array}$} & $\begin{array}{l}\text { Less than } 200,000 \\
\text { won }\end{array}$ & $67(11.8 \%)$ \\
\hline & $\begin{array}{l}200,000 \sim \text { less than } \\
500,000 \text { won }\end{array}$ & $353(62.0 \%)$ \\
\hline & $\begin{array}{l}500,000 \text { won and } \\
\text { more }\end{array}$ & $149(26.2 \%)$ \\
\hline \multirow{5}{*}{$\begin{array}{l}\text { The first time } \\
\text { to begin } \\
\text { gambling }\end{array}$} & No gambling & $247(43.1 \%)$ \\
\hline & Elementary school & $61(10.6 \%)$ \\
\hline & Middle school & $73(12.7 \%)$ \\
\hline & High school & $75(13.1 \%)$ \\
\hline & College & $117(20.4 \%)$ \\
\hline \multirow{2}{*}{$\begin{array}{l}\text { Illegal } \\
\text { gambling } \\
\text { experience }\end{array}$} & Yes & $35(6.1 \%)$ \\
\hline & No & $542(93.9 \%)$ \\
\hline \multirow{3}{*}{$\begin{array}{l}\text { Route to start } \\
\text { gambling }\end{array}$} & $\begin{array}{l}\text { Suggestion from } \\
\text { family }\end{array}$ & $49(15.6 \%)$ \\
\hline & $\begin{array}{l}\text { Suggestion from a } \\
\text { friend }\end{array}$ & $139(44.1 \%)$ \\
\hline & $\begin{array}{l}\text { By mass media or } \\
\text { internet }\end{array}$ & $127(40.3 \%)$ \\
\hline
\end{tabular}

\section{B. Types of gambling activity}

Table 2 describes the types of gambling. The most prevalent gambling activity was lottery purchase and the rate of the respondents who experienced that activity was $35.4 \%$. 
The rates of the respondents who experienced fellowship games such as 'hwatu' or cards, entertainment online games with cash or cyber money, sports promotion lottery tickets such as sports toto, illegal private gambling, horse racing betting, casino betting, bullfight betting, bicycle racing betting, and boat racing betting were $32.8 \%, 21.0 \%, 6.1 \%$, $6.1 \%, 3.3 \%, 2.4 \%, 0.9 \%, 0.3 \%$, and $0.3 \%$ respectively.

Table- II: Prevalent types of gambling activity $(n=577)$

\begin{tabular}{|c|c|c|c|}
\hline Description & Yes & No & Total \\
\hline Lottery purchase & $\begin{array}{c}204 \\
(35.4 \%) \\
\end{array}$ & $\begin{array}{c}373 \\
(64.6 \%) \\
\end{array}$ & $\begin{array}{c}577 \\
(100.0 \%) \\
\end{array}$ \\
\hline Fellowship games & $\begin{array}{c}189 \\
(32.8 \%) \\
\end{array}$ & $\begin{array}{c}388 \\
(67.2 \%) \\
\end{array}$ & $\begin{array}{c}577 \\
(100.0 \%) \\
\end{array}$ \\
\hline $\begin{array}{l}\text { Entertainment } \\
\text { online games }\end{array}$ & $\begin{array}{c}121 \\
(21.0 \%)\end{array}$ & $\begin{array}{c}456 \\
(79.0 \%)\end{array}$ & $\begin{array}{c}577 \\
(100.0 \%)\end{array}$ \\
\hline Sports toto & $\begin{array}{c}35 \\
(6.1 \%) \\
\end{array}$ & $\begin{array}{c}542 \\
(93.9 \%)\end{array}$ & $\begin{array}{c}577 \\
(100.0 \%)\end{array}$ \\
\hline $\begin{array}{l}\text { Illegal private } \\
\text { gambling* }\end{array}$ & $\begin{array}{c}35 \\
(6.1 \%)\end{array}$ & $\begin{array}{c}542 \\
(93.9 \%)\end{array}$ & $\begin{array}{c}577 \\
(100.0 \%)\end{array}$ \\
\hline $\begin{array}{l}\text { Horse racing } \\
\text { betting }\end{array}$ & $\begin{array}{c}19 \\
(3.3 \%) \\
\end{array}$ & $\begin{array}{c}558 \\
(96.7 \%) \\
\end{array}$ & $\begin{array}{c}577 \\
(100.0 \%) \\
\end{array}$ \\
\hline Casino betting & $\begin{array}{c}14 \\
(2.4 \%)\end{array}$ & $\begin{array}{c}563 \\
(97.6 \%)\end{array}$ & $\begin{array}{c}577 \\
(100.0 \%)\end{array}$ \\
\hline Bullfight betting & $\begin{array}{c}5 \\
(0.9 \%) \\
\end{array}$ & $\begin{array}{c}572 \\
(99.1 \%) \\
\end{array}$ & $\begin{array}{c}577 \\
(100.0 \%) \\
\end{array}$ \\
\hline $\begin{array}{l}\text { Bicycle racing } \\
\text { betting }\end{array}$ & $\begin{array}{c}2 \\
(0.3 \%)\end{array}$ & $\begin{array}{c}575 \\
(99.7 \%)\end{array}$ & $\begin{array}{c}577 \\
(100.0 \%)\end{array}$ \\
\hline Boat racing betting & 2 & 575 & 577 \\
\hline
\end{tabular}

\begin{tabular}{l|l|l|l}
\hline & $(0.3 \%)$ & $(99.7 \%)$ & $(100.0 \%)$ \\
\hline
\end{tabular}

*: Except 'illegal private gambling,' others are all legally permitted gambling

\section{C.Classification of gamblers by sub-type of CPGI}

Classification of gamblers by sub-type of CPGI are described as seen in Table 3. Non-problem, low risk, moderate risk, and problem gamblers were $69.7 \%, 16.3 \%$, $8.8 \%$, and $5.2 \%$ respectively. So, the prevalence rate of gambling addiction, which is the total rates of moderate risk and problem gamblers was $14.0 \%$. The figure is higher than $11.1 \%$ of college students in 2011 which was investigated by Kwon [5].

Table- III: Gamblers by sub-type of CPGI $(n=577)$

\begin{tabular}{c|c}
\hline Description & $\mathrm{N}(\%)$ \\
\hline Non-problem & $402(69.7 \%)$ \\
\hline Low risk & $94(16.3 \%)$ \\
\hline Moderate risk & $51(8.8 \%)$ \\
\hline Problem & $30(5.2 \%)$ \\
\hline Total & $577(100.0 \%)$ \\
\hline
\end{tabular}

\section{D.General characteristics of gamblers by sub-types}

The general characteristics of gamblers by sub-types are described as seen in Table 4. There was significant statistical difference between sub-types in gender, college grade, spending money, the first time to begin gambling, illegal gambling experience, and route to start gambling.

Table- IV: General characteristics of gamblers by sub-types $(n=577)$

\begin{tabular}{|c|c|c|c|c|c|c|c|c|}
\hline \multirow{2}{*}{\multicolumn{2}{|c|}{ Variables }} & \multicolumn{5}{|c|}{ Sub-types of gambler } & \multirow[t]{2}{*}{$x^{2}$} & \multirow[t]{2}{*}{$p$} \\
\hline & & Non-problem & Low risk & Moderate risk & Problem & Total $(\%)$ & & \\
\hline \multirow[t]{2}{*}{ Gender } & Male & $\begin{array}{c}156 \\
(59.3 \%)\end{array}$ & $\begin{array}{c}53 \\
(20.2 \%)\end{array}$ & $\begin{array}{c}35 \\
(13.3 \%)\end{array}$ & $\begin{array}{c}19 \\
(7.2 \%)\end{array}$ & $\begin{array}{c}263 \\
(100.0 \%)\end{array}$ & \multirow{2}{*}{26.593} & \multirow{2}{*}{$<.001$} \\
\hline & Female & $\begin{array}{c}246 \\
(78.3 \%) \\
\end{array}$ & $\begin{array}{c}41 \\
(13.1 \%) \\
\end{array}$ & $\begin{array}{c}16 \\
(5.1 \%) \\
\end{array}$ & $\begin{array}{c}11 \\
(3.5 \%) \\
\end{array}$ & $\begin{array}{c}314 \\
(100.0 \%) \\
\end{array}$ & & \\
\hline \multirow[t]{4}{*}{$\begin{array}{l}\text { College } \\
\text { grade }\end{array}$} & Freshman & $\begin{array}{c}147 \\
(70.0 \%)\end{array}$ & $\begin{array}{c}37 \\
(17.6 \%)\end{array}$ & $\begin{array}{c}20 \\
(9.5 \%)\end{array}$ & $\begin{array}{c}6 \\
(2.9 \%)\end{array}$ & $\begin{array}{c}210 \\
(100.0 \%)\end{array}$ & \multirow{4}{*}{17.336} & \multirow{4}{*}{.044} \\
\hline & 2nd grade & $\begin{array}{c}132 \\
(73.3 \%)\end{array}$ & $\begin{array}{c}25 \\
(13.9 \%)\end{array}$ & $\begin{array}{c}17 \\
(9.4 \%)\end{array}$ & $\begin{array}{c}6 \\
(3.3 \%)\end{array}$ & $\begin{array}{c}180 \\
(100.0 \%)\end{array}$ & & \\
\hline & 3rd grade & $\begin{array}{c}77 \\
(71.3 \%) \\
\end{array}$ & $\begin{array}{c}17 \\
(15.7 \%) \\
\end{array}$ & $\begin{array}{c}5 \\
(4.6 \%) \\
\end{array}$ & $\begin{array}{c}9 \\
(8.3 \%) \\
\end{array}$ & $\begin{array}{c}108 \\
(100.0 \%) \\
\end{array}$ & & \\
\hline & 4 th grade & $\begin{array}{c}46 \\
(58.2 \%) \\
\end{array}$ & $\begin{array}{c}15 \\
(19.0 \%) \\
\end{array}$ & $\begin{array}{c}9 \\
(11.4 \%) \\
\end{array}$ & $\begin{array}{c}9 \\
(11.4 \%) \\
\end{array}$ & $\begin{array}{c}79 \\
(100.0 \%) \\
\end{array}$ & & \\
\hline \multirow{3}{*}{$\begin{array}{l}\text { Spending } \\
\text { money per } \\
\text { month } \\
\text { (won) }\end{array}$} & $\begin{array}{l}\text { Less than } \\
200,000\end{array}$ & $\begin{array}{c}52 \\
(77.6 \%) \\
\end{array}$ & $\begin{array}{c}7 \\
(10.4 \%) \\
\end{array}$ & $\begin{array}{c}6 \\
(9.0 \%) \\
\end{array}$ & $\begin{array}{c}2 \\
(3.0 \%) \\
\end{array}$ & $\begin{array}{c}67 \\
(100.0 \%) \\
\end{array}$ & \multirow{3}{*}{15.570} & \multirow{3}{*}{.016} \\
\hline & $\begin{array}{l}200,000 \sim \text { les } \\
\text { s than } \\
500,000\end{array}$ & $\begin{array}{c}258 \\
(73.1 \%)\end{array}$ & $\begin{array}{c}49 \\
(13.9 \%)\end{array}$ & $\begin{array}{c}27 \\
(7.6 \%)\end{array}$ & $\begin{array}{c}19 \\
(5.4 \%)\end{array}$ & $\begin{array}{c}353 \\
(100.0 \%)\end{array}$ & & \\
\hline & $\begin{array}{l}500,000 \text { and } \\
\text { more }\end{array}$ & $\begin{array}{c}87 \\
(58.4 \%) \\
\end{array}$ & $\begin{array}{c}37 \\
(24.8 \%) \\
\end{array}$ & $\begin{array}{c}17 \\
(11.4 \%)\end{array}$ & $\begin{array}{c}8 \\
(5.4 \%)\end{array}$ & $\begin{array}{c}149 \\
(100.0 \%)\end{array}$ & & \\
\hline \multirow{4}{*}{$\begin{array}{l}\text { The first } \\
\text { time to } \\
\text { begin } \\
\text { gambling }\end{array}$} & $\begin{array}{l}\text { Elementary } \\
\text { school }\end{array}$ & $\begin{array}{c}32 \\
(52.5 \%) \\
\end{array}$ & $\begin{array}{c}15 \\
(24.6 \%) \\
\end{array}$ & $\begin{array}{c}7 \\
(11.5 \%) \\
\end{array}$ & $\begin{array}{c}7 \\
(11.5 \%) \\
\end{array}$ & $\begin{array}{c}61 \\
(100.0 \%) \\
\end{array}$ & \multirow{4}{*}{17.841} & \multirow{4}{*}{.037} \\
\hline & $\begin{array}{l}\text { Middle } \\
\text { school }\end{array}$ & $\begin{array}{c}37 \\
(50.7 \%) \\
\end{array}$ & $\begin{array}{c}19 \\
(26.0 \%) \\
\end{array}$ & $\begin{array}{c}9 \\
(12.3 \%) \\
\end{array}$ & $\begin{array}{c}8 \\
(11.0 \%) \\
\end{array}$ & $\begin{array}{c}73 \\
(100.0 \%) \\
\end{array}$ & & \\
\hline & High school & $\begin{array}{c}31 \\
(41.3 \%) \\
\end{array}$ & $\begin{array}{c}20 \\
(26.7 \%) \\
\end{array}$ & $\begin{array}{c}20 \\
(26.7 \%) \\
\end{array}$ & $\begin{array}{c}4 \\
(5.3 \%) \\
\end{array}$ & $\begin{array}{c}75 \\
(100.0 \%) \\
\end{array}$ & & \\
\hline & College & $\begin{array}{c}74 \\
(63.2 \%)\end{array}$ & $\begin{array}{c}21 \\
(17.9 \%)\end{array}$ & $\begin{array}{c}12 \\
(10.3 \%)\end{array}$ & $\begin{array}{c}10 \\
(8.5 \%)\end{array}$ & $\begin{array}{c}117 \\
(100.0 \%)\end{array}$ & & \\
\hline
\end{tabular}




\begin{tabular}{|c|c|c|c|c|c|c|c|c|}
\hline \multirow{2}{*}{$\begin{array}{l}\text { Illegal } \\
\text { gambling } \\
\text { experience }\end{array}$} & Yes & $\begin{array}{c}7 \\
(20.0 \%) \\
\end{array}$ & $\begin{array}{c}6 \\
(17.1 \%) \\
\end{array}$ & $\begin{array}{c}13 \\
(37.1 \%) \\
\end{array}$ & $\begin{array}{c}9 \\
(25.7 \%) \\
\end{array}$ & $\begin{array}{c}35 \\
(100.0 \%) \\
\end{array}$ & \multirow{2}{*}{77.143} & \multirow{2}{*}{$<.001$} \\
\hline & No & $\begin{array}{c}395 \\
(72.9 \%)\end{array}$ & $\begin{array}{c}88 \\
(16.2 \%)\end{array}$ & $\begin{array}{c}38 \\
(7.0 \%)\end{array}$ & $\begin{array}{c}21 \\
(3.9 \%)\end{array}$ & $\begin{array}{c}542 \\
(100.0 \%)\end{array}$ & & \\
\hline \multirow{3}{*}{$\begin{array}{l}\text { Route to } \\
\text { start } \\
\text { gambling }\end{array}$} & $\begin{array}{l}\text { Suggestion } \\
\text { from family }\end{array}$ & $\begin{array}{c}25 \\
(51.0 \%) \\
\end{array}$ & $\begin{array}{c}17 \\
(34.7 \%) \\
\end{array}$ & $\begin{array}{c}4 \\
(8.2 \%) \\
\end{array}$ & $\begin{array}{c}3 \\
(6.1 \%) \\
\end{array}$ & $\begin{array}{c}49 \\
(100.0 \%) \\
\end{array}$ & \multirow{3}{*}{19.228} & \multirow{3}{*}{.004} \\
\hline & $\begin{array}{l}\text { Suggestion } \\
\text { from a friend }\end{array}$ & $\begin{array}{c}58 \\
(41.7 \%) \\
\end{array}$ & $\begin{array}{c}35 \\
(25.2 \%) \\
\end{array}$ & $\begin{array}{c}28 \\
(20.1 \%) \\
\end{array}$ & $\begin{array}{c}18 \\
(12.9 \%) \\
\end{array}$ & $\begin{array}{c}139 \\
(100.0 \%) \\
\end{array}$ & & \\
\hline & $\begin{array}{l}\text { By mass } \\
\text { media or the } \\
\text { internet }\end{array}$ & $\begin{array}{c}81 \\
(63.8 \%)\end{array}$ & $\begin{array}{c}22 \\
(17.3 \%)\end{array}$ & $\begin{array}{c}16 \\
(12.6 \%)\end{array}$ & $\begin{array}{c}8 \\
(6.3 \%)\end{array}$ & $\begin{array}{c}127 \\
(100.0 \%)\end{array}$ & & \\
\hline
\end{tabular}

\section{E.Happiness, self-esteem, impulsivity, and irrational gambling belief of gambler sub-types}

A relationship between the following variables and gambler sub-types is described as seen in Table 5. A significant statistical difference was found between sub-types in self-esteem, impulsivity, and irrational gambling belief. Low risk gamblers had higher self-esteem than non-problem gamblers. Moderate risk gamblers had higher impulsivity than non-problem or low risk gamblers. Moderate or problem gamblers had significantly higher irrational gambling belief than non-problem or low risk gamblers.

Table- V: Happiness, self-esteem, impulsivity, irrational gambling belief of gambler sub-types $(n=577)$

\begin{tabular}{|c|c|c|c|c|c|c|}
\hline \multicolumn{3}{|c|}{ Variables } & $\begin{array}{c}\text { Happines } \\
\mathrm{s}\end{array}$ & $\begin{array}{l}\text { Self-es } \\
\text { teem }\end{array}$ & $\begin{array}{l}\text { Impulsi } \\
\text { vity }\end{array}$ & $\begin{array}{l}\text { Irrational } \\
\text { gambling } \\
\text { belief }\end{array}$ \\
\hline \multicolumn{3}{|c|}{ Range } & $1 \sim 7$ & $1 \sim 4$ & $1 \sim 4$ & $1 \sim 5$ \\
\hline \multirow{8}{*}{$\begin{array}{l}\text { Sub-type } \\
\text { s of } \\
\text { gambler }\end{array}$} & \multirow{2}{*}{$\begin{array}{l}\text { Non-gam } \\
\text { bler }\end{array}$} & Mean & 5.04 & $2.89^{\mathrm{a}}$ & $1.99^{\mathrm{a}}$ & $1.84^{\mathrm{a}}$ \\
\hline & & SD & 1.23 & 0.52 & 0.57 & 0.84 \\
\hline & \multirow{2}{*}{ Low risk } & Mean & 5.08 & $3.08^{\mathrm{b}}$ & $1.97^{\mathrm{b}}$ & $2.08^{\mathrm{b}}$ \\
\hline & & SD & 0.94 & 0.47 & 0.54 & 0.78 \\
\hline & \multirow{2}{*}{$\begin{array}{l}\text { Moderate } \\
\text { risk }\end{array}$} & Mean & 4.84 & $2.95^{\mathrm{c}}$ & $2.28^{\mathrm{c}}$ & $2.77^{\mathrm{c}}$ \\
\hline & & SD & 1.15 & 0.57 & 0.53 & 0.72 \\
\hline & \multirow{2}{*}{ Problem } & Mean & 4.47 & $2.82^{\mathrm{d}}$ & $2.21^{\mathrm{d}}$ & $2.98^{\mathrm{d}}$ \\
\hline & & SD & 1.55 & 0.47 & 0.52 & 0.76 \\
\hline \multicolumn{3}{|l|}{$\mathrm{F}$} & 2.290 & 3.639 & 5.304 & 34.128 \\
\hline \multicolumn{3}{|l|}{$p$} & .077 & $.013^{\mathrm{b}>\mathrm{a}}$ & $.001^{\mathrm{c} \mathrm{a}, \mathrm{b}}$ & $<.001^{\mathrm{c}, \mathrm{d}>\mathrm{a}, \mathrm{b}}$ \\
\hline
\end{tabular}

\section{F. Matters concerning the required support for gamblers}

When a gambling problem occurred, the number of respondents who knew a free counselling center was only 20 $(3.5 \%)$, so it is quite required to carry out preventive education and to publicize free counselling centers for a gambling problem. The support needed when there is a gambling problem was investigated as seen in Table 6. 'Psychotherapy and counseling' was 43.2\%, 'family counseling' was $22.0 \%$, 'hospital treatment' was $17.7 \%$, 'group therapy or GA meeting' was $8.7 \%$, and 'financial and legal consultation' was $8.5 \%$.

Table- VI: Matters concerning the required support for

\begin{tabular}{c|l|c}
\hline \multicolumn{3}{c}{ gamblers $(\mathrm{n}=577)$} \\
\hline \multicolumn{1}{c|}{ Item } & \multicolumn{1}{|c}{ Category } & $\mathbf{N}(\%)$ \\
\hline \multirow{2}{*}{$\begin{array}{c}\text { Awareness of free } \\
\text { counselling center }\end{array}$} & Yes & $20(3.5 \%)$ \\
\cline { 2 - 3 } & No & $557(96.5 \%)$ \\
\hline \multirow{4}{*}{ Required support* } & Psychotherapy and counseling & $334(43.2 \%)$ \\
\cline { 2 - 3 } & Family counseling & $170(22.0 \%)$ \\
\cline { 2 - 3 } & Hospital treatment & $137(17.7 \%)$ \\
\cline { 2 - 3 } & Group therapy or GA meeting & $67(8.7 \%)$ \\
\cline { 2 - 3 } & Financial and legal consultation & $66(8.5 \%)$ \\
\hline
\end{tabular}

\section{DISCUSSION}

This study aims to investigate a seriousness of gambling problems among college students in Korea and a relationship between several variables and gambler sub-type of CPGI. When a level of gambling was classified into gambler sub-types of CPGI in this study, Non-problem, low risk, moderate risk, and problem gamblers were $69.7 \%, 16.3 \%$, $8.8 \%$, and $5.2 \%$ respectively. The prevalence rate of gambling addiction, which is the total rates of moderate risk and problem gamblers was $14.0 \%$. It is partly consistent with the study that gambling problems are most likely to occur during college years [17]. Kwon reported that the rate of college students in 2011 was $11.1 \%$ in Korea [5]. In case of other country, Mubarak and Blanksby reported that the prevalence rate was estimated $11.7 \%$, specifically $8.6 \%$ for problem gambling and $3.1 \%$ for probable pathological gambling among college students [18]. Meanwhile, the prevalence rates of gambling addiction in Korean adults were $7.2 \%$ in $2012,5.4 \%$ in 2014 , and $5.1 \%$ in 2016 [3]. The gambling prevalence rate among college students is high in comparison to the adult population and the gambling prevalence rate among college students seems to be rapidly increasing in Korea.

The current study reported that there was a significant statistical difference between gambler sub-types in gender. The prevalence rates of gambling addiction were $20.5 \%$ among male students and $8.6 \%$ among female students. There are other studies which identified male students are more serious than female students in a gambling problem [19-22]. Huang et al. identified $4.3 \%$ of male students and $0.4 \%$ of female students as problem or pathological gamblers in the USA [19]. Leppink et al. estimated that approximately 5 9\% of male college students and 1 2\% of female college students are problem and pathologic gamblers in North America [20]. Male college students tend to pay more attention to 
sports and games than female college students, it seems to lead to the frequent occurrence of betting activities among male college students.

In the impulsivity, moderate gamblers were higher than non-problem or low risk gamblers. This is consistent with the previous studies [21,22]. Problem gamblers are prone to impulse control disorders [21]. A study also showed the high impulsivity scores significantly associated with severity of gambling pathology [20]. In urban male adolescent study, males with high impulsivity were on average twice as likely to be at risk or problem gamblers as those with low impulsivity [23]. All of this is because people with high impulsivity tend to make haste decisions without fully considering the potential negative results [23].

In the irrational gambling belief, moderate or problem gamblers were significantly higher than non-problem or low risk gamblers. This current result is also partly consistent with the previous studies [24]. The irrational belief such as a confidence that he can win without any reasonable foundation might encourage gambling activity [25]. In particular, the early 'big win' of gambling career increases unrealistic expectations for future profits, and also affects selective memory enhancement [25].

Most gambling counseling centers, including the Korea Center on Gambling Problems, are operated free of charge. However, the number of respondents who knew free counselling centers for a gambling problem was only 20 students $(3.5 \%)$, so it is quite required to publicize free counselling centers through preventive education or other opportunities. The support needed for gamblers was investigated mainly as 'psychotherapy and counseling (43.2\%)', 'family counseling (22.0\%)' and 'hospital treatment (17.7\%)'. Many previous references also suggested that problem gamblers needed both addiction counseling and mental health care [2].

A survey on gambling problem by gambler sub-types of CPGI among 577 Korean college students shows that a tendency of gambling activity is generally the same as previous research, but there are slight differences in detail, so, repeated future studies are still necessary. As the spread of the internet, the activation of the game industry, and the economic downturn in the long term increase problem gamblers of college students in Korea, it is important to grasp the actual situation and preventive approach to the gambling addiction risk factor seems to be an effective strategy.

\section{CONCLUSION}

The study aims to survey a seriousness of gambling problems of college students and investigate a relationship between several variables and gambling problems. The total percentage of moderate risk and problem gamblers was $14.0 \%$. The impulsivity of moderate risk gamblers were higher than that of non-problem or low risk gamblers. The irrational gambling belief of moderate or problem gamblers were significantly higher than that of non-problem or low risk gamblers. It is required to continue future surveys on a gambling problem among college students and to take a proper measure preventing a gambling problem.

\section{CONFLICT OF INTEREST}

We declare no conflict of interest.

\section{ACKNOWLEDGMENT}

This work was supported by National Research Foundation of Korea (NRF-2017R1D1A1B 03028538).

\section{REFERENCES}

1. J. Ferris and H. Wynne, The Canadian Problem Gambling Index: final report. Canadian Centre on Substance Abuse, 2001 http://www.ccgr.ca/en/projects/resources/CPGI-Final-Report-English.p df

2. G. J. Smith and H, J, Wynne, Measuring Gambling and Problem Gambling in Alberta Using the Canadian Problem Gambling Index (CPGI): final report. Alberta Gaming Research Institute, 2002. http://dx.doi.org/10.11575/PRISM/9890

3. Gallup Korea Research Institute, A Survey on Actual State of Use in Gambling Industry: 2016. National Gambling Control Commission, 2016.

http://dl.nanet.go.kr/SearchDetailView.do?cn=MONO1201642969\&sy sid=uci

4. Z. Szczyrba, V. Mravčík, D. Fiedor, J. Černý, and I. Smolová, "Gambling in the Czech Republic," Addiction, 110(7), 2015, pp. 1076-1081. https://doi.org/10.1111/add.12884

5. B. S. Kwon and Y.H. Kim, "A study of gambling addiction and its actual conditions among university students in Korea," Mental Health and Social Work, 39, 2011, pp. 5-28 http://www.riss.kr.proxy.nsu.ac.kr:8010/link?id=A101148464

6. American Psychiatric Association, Diagnostic and Statistical Manual of Mental Disorders. Fourth Edition Text Revision (DSM-IV-TR), American Psychiatric Association Press, 2000.

7. H. P. Lee, J. G. Lee, H. W. Kim, T. W. Kim, Y. O. Han, and S. K. Lee, Pascal's Betting, Temptation of Gambling - Understanding and Treatment of Gambling. Hakjisa, 2013 http://dl.nanet.go.kr/SearchDetailView.do?cn=MONO1201302176\&sy sid=uci

8. H. W. Kim, Why Do We Fall into Gambling. Soulmate, 2013.

9. Z. Steel and A. Blaszczynski, "Impulsivity, personality disorders and pathological gambling severity," Addiction, 93(6), 1998, pp. 895-905. https://doi.org/10.1046/j.1360-0443.1998.93689511.x

10. R. Stinchfield, W. Hanson, and D. Olson, "Problem and pathological gambling among college students," New Directions for Student Services, (113), 2006, pp. 63-72. https://doi.org/10.1002/ss.196

11. S. Lyubomirsky and H. S. Lepper, "A measure of subjective happiness preliminary reliability and construct validation," Social Indicators Research, 46(2), 1999, pp. 137-155. https://doi.org/10.1023/A:1006824100041

12. M. Rosenberg, Society and the Adolescent Self-image. Princeton University Press, 1965.

13. D. G. Kim and H. N. Ahn, "A validation study of NEO personality assessment system(NEO-PAS) for adolescents," The Korea Journal of Youth Counseling, (1), 2006, pp. 77-91 http://www.riss.kr.proxy.nsu.ac.kr:8010/link?id=A102573769

14. S. J. Kwon, K. H. Kim, and J. O. Choi, "Awareness of adult gambling and predictors of gambling behavior in children," Korean Journal of Health Psychology, 11(1), 2006, pp. 147-162. http://www.dbpia.co.kr/Article/NODE06368645

15. H. P. Lee, "The Relationship of Irrational Gambling Belief, Gambling Motive, and Risk Taking with Pathological Gambling," Doctoral Thesis, Korea University, 2003.

16. T. A. Steenbergh, A. W. Meyers, R. K. May, and J. P. Whelan, "A self-report measure of gambler's maladaptive beliefs: Initial psychometric properties," Journal of Addictive Behaviors, 16(2), 2002, pp. 143-149.

17. H. J. Shaffer, M. N. Hall, and B. Vander, "Estimating the prevalence of disordered gambling behavior in the United States and Canada: a research synthesis," American Journal of Public Health, 89(9), 1999, pp 1369-1376. DOI: 10.2105/AJPH.89.9.1369 
18. A. Mubarak and P. Blanksby, "A study on problem and pathological gambling among university students in South Australia," Journal of Higher Education Policy and Management, 35(5), 2013, pp. 471-482. https://doi.org/10.1080/1360080X.2013.775927

19. J. Huang, D. Jacobs, J. Derevensky, R. Gupta, and T. Paskus, "A national study on gambling among US college student-athletes," Journal of American College Health, 56(2), 2007, pp. 93-99. https://doi.org/10.3200/JACH.56.2.93-100

20. E. W. Leppink, S. A. Redden, and J. E. Grant, "Impulsivity and gambling: a complex clinical association across three measures," American Journal on Addictions, 25(2), 2016, pp. 138-144. https://doi.org/10.1111/ajad.12341

21. S. Singh, G. K. Mallaram, and S. Sarkar, "Pathological gambling: an overview," Medical Journal of Dr. D.Y. Patil Vidyapeeth, 10(2), 2017, pp. 120-127. DOI: 10.4103/0975-2870.202105

22. D. Cunha, B. Sousa, and A. P. Relvas, "Risk factors for pathological gambling along a continuum of severity: Individual and relational variables," Journal of Gambling Issues, (35), 2017, pp. 49-73. http://igi.camh.net/doi/pdf/10.4309/jgi.2017.35.3

23. W. Liu, G. P. Lee, A. Goldweber, H. Petras, C. L. Storr, N. S. Ialongo, and S. S. Martins, "Impulsivity trajectories and gambling in adolescence among urban male youth," Addiction, 108(4), 2013, pp. 780-788. https://doi.org/10.1111/add.12049

24. S. J. Kwon, K. H. Kim, H. G. Seong, M. K. Rhee, and S. G. Kang, "Illegal internet gambling: problems, risk factors, and prevention strategies," The Korean Journal of Health Psychology, 12(1), 2007, pp. 1-19. http://www.riss.kr.proxy.nsu.ac.kr:8010/link?id=A100631865

25. I. Lund, "Irrational beliefs revisited: exploring the role of gambling preferences in the development of misconceptions in gamblers," Addiction Research and Theory, 19(1), 2011, pp. 40-46. https://doi.org/10.3109/16066359.2010.493979

\section{AUTHORS PROFILE}

Seong Ui Kim is a professor of Saekyung university college in Korea. She obtained doctoral degree at Soonchunhyang University in Korea. Her specialty is mental health care nursing. She is interested in health promotion and hospice education.

Jung Hyun Choi is a professor of Namseoul university in Korea. She obtained doctoral degree at Catholic University in Korea. Her specialty is community health nursing. She is interested in health promotion and health education. She published some researches about gambling addiction and gambling abstinence self-efficacy.

Jum Mi Park is a professor of Namseoul university in Korea. She obtained doctoral degree at Hanyang University in Korea. Her specialty is women health nursing. She published some researches about infertility and abortion. 